Rabaska

Revue d'ethnologie de l'Amérique française

\title{
Encyclopédie du patrimoine culturel de l'Amérique française
}

\section{Martin Fournier}

Volume 7, 2009

URI : https://id.erudit.org/iderudit/038344ar

DOI : https://doi.org/10.7202/038344ar

Aller au sommaire du numéro

Éditeur(s)

Société québécoise d'ethnologie

ISSN

1703-7433 (imprimé)

1916-7350 (numérique)

Découvrir la revue

Citer ce document

Fournier, M. (2009). Encyclopédie du patrimoine culturel de l'Amérique

française. Rabaska, 7, 127-134. https://doi.org/10.7202/038344ar d'utilisation que vous pouvez consulter en ligne.

https://apropos.erudit.org/fr/usagers/politique-dutilisation/ 


\section{Colloques et rassemblements}

\section{Encyclopédie du patrimoine culturel de l'Amérique française}

MARTIn Fournier Université Laval

Un atelier de réflexion a eu lieu le $1^{\text {er }}$ décembre 2008 à Québec, afin de faire le point sur l'état d'avancement de l'Encyclopédie du patrimoine culturel de l'Amérique française et de réfléchir aux améliorations qu'il serait bienvenu d'apporter à l'ouvrage au cours de l'année 2009. Cet atelier réunissait 17 personnes, dont les membres du comité scientifique Pierre Anctil (Université d'Ottawa), André Charbonneau (Parcs Canada), Leslie Choquette (Assomption College, Massachussets), Yves Laberge (Université Laval), Jacques Mathieu (Université Laval) et Pierre-Yves Mocquais (Université de Calgary), le président du comité de direction Marcel Masse (ministre retraité), le codirecteur du projet Yves Bergeron (Université du Québec à Montréal), le coordonnateur du projet Martin Fournier, les adjoints à la coordination François-X. Côté et Catherine Ferland, le coordonnateur régional au Manitoba Luc Côté (Collège universitaire de Saint-Boniface), ainsi que les consultants externes Mario Asselin (firme Opossum de Québec), Aline Carpentier-Lecorre (archives de Charentes-Maritimes à La Rochelle), Pierre Guillaume (Université de Bordeaux 3), Nathalie Hamel (Commission des biens culturels du Québec) et Gilles Herman (Éditions du Septentrion de Québec). Exceptionnellement, le directeur de l'Encyclopédie, Laurier Turgeon (Université Laval), n'a pu assister à la rencontre étant en mission à Haïti pour contribuer à l'identification, à la préservation et à la mise en valeur du patrimoine immatériel haïtien. Laurier Turgeon a néanmoins présenté une communication vidéo enregistrée à l'avance sur le thème de la patrimonialisation.

\section{État de l'Encyclopédie au $1^{\text {er }}$ décembre 2008}

L'Encyclopédie du patrimoine culturel de l'Amérique française est à ses débuts un ouvrage qui est diffusé exclusivement sur la toile. Le site 
Www.ameriquefrancaise.org a été inauguré en avril 2008 et lancé officiellement à Paris et à Québec en avril et en juin 2008. À la date de l'atelier, l'Encyclopédie comprend 50 articles en ligne, accompagnés de 1300 documents multimédias (visuels, sonores, audiovisuels et textuels). L'Encyclopédie compte également un module interactif destiné à la clientèle des 13-16 ans portant sur la traite de fourrures au Canada de 1650 à 1850, et au patrimoine relié à cette activité fondatrice.

La fréquentation du site est en hausse constante depuis l'inauguration et se chiffre à 4000 visiteurs en novembre 2008. La réalisation de l'Encyclopédie repose sur un important réseau pancanadien de partenaires, développé au cours des deux années précédentes, afin d'appuyer l'identification des sujets, la rédaction et l'illustration des articles sur les communautés francophones de toutes les régions du Canada. L'Encyclopédie compte également sur de nombreux collaborateurs en France et aux États-Unis. Les principaux partenaires actuels de l'Encyclopédie sont les suivants: Université Laval (base du projet), l'Université Sainte-Anne, l'Université de Moncton, l'Université de Sudbury, le Collège universitaire de Saint-Boniface, l'Université de Régina, la Bibliothèque et les Archives nationales du Québec, le Musée national des beaux-arts du Québec, Parcs Canada, l'Office national du film du Canada, le Consulat général de la France à Québec, et autres.

\section{Les principaux objectifs de l'année 2009}

D'ici le mois de juin 2009, l'équipe de production prévoit mettre en ligne 65 nouveaux articles, accompagnés d'environ 1500 documents multimédias, ainsi qu'un module interactif supplémentaire destiné aux 13-16 ans portant cette fois sur la chanson d'expression française au Canada. L'Encyclopédie inaugurera également son volet en langue anglaise avec la traduction d'une quarantaine d'articles déjà publiés en langue française. Le recrutement de nouveaux partenaires canadiens est également amorcé, incluant principalement le Centre de la francophonie des Amériques, la Société des établissements de plein-air du Québec, le Musée québécois de culture populaire, le Musée acadien de l'Université de Moncton. La rédaction d'articles en provenance de France sera également mieux structurée sous la supervision d'Aline Carpentier-Lecorre. Enfin, des démarches de financement sont prévues afin d'assurer la production de 100 articles supplémentaires en 2010.

\section{Déroulement de l'atelier}

I- Premier sujet de discussion : exploiter davantage le potentiel d'Internet Présentation de François-X. Côté pour 1'Encyclopédie, suivie des 
commentaires de l'expert invité Mario Asselin, directeur de la firme informatique Opossum, apprentissage et technologies.

A) François-X. Côté explique la nécessité dans laquelle se trouve l'Encyclopédie de migrer vers une plateforme informatique plus performante afin de dynamiser et de clarifier son utilisation par les internautes, de favoriser l'accès aux contenus et d'afficher davantage le caractère multimédia de l'ouvrage. Il présente la nouvelle page d'accueil en projet et la page d'accès détaillée aux contenus, ainsi que les principales fonctions prévues. Pour validation et bonification par le groupe de discussion.

B) Mario Asselin expose quelques données sur les usagers de la Toile et leurs diverses façons de l'utiliser, notamment en fonction de l'âge. Le principal enjeu est celui de l'interactivité, ou de la participation des usagers. À ses yeux, l'Encyclopédie doit rechercher le juste milieu entre la consultation des contenus (clientèle plus âgée) et la création des contenus (clientèle plus jeune) dans des sections de l'Encyclopédie ou sous une forme contrôlée (en écartant la création collaborative par tous comme dans Wikipédia). Il ressort également de son exposé que la Toile est un médium en évolution rapide et que le souci de l'Encyclopédie de rester à l'affût des développements en ce domaine est pertinent.

La discussion qui suit met l'accent sur le principal objectif de l'Encyclopédie, qui consiste en la création d'un excellent contenu sur le patrimoine de l'Amérique française. À partir de ce contenu de bonne tenue scientifique, appuyé par une abondante documentation multimédia, l'enjeu est de faire réagir les internautes et de leur donner la possibilité d'interagir sans intervenir directement dans le contenu des articles. Les contenus doivent être présentés de façon exhaustive et détaillée, comme on le fait habituellement dans les encyclopédies. Il serait bon aussi de prévoir de brèves introductions sur certains sujets, thèmes ou concepts, pour mieux cheminer dans l'Encyclopédie et bien comprendre les contextes historiques, culturels ou géographiques des divers sujets exposés dans les articles.

On rappelle que le concept de patrimoine évolue, que les objets patrimoniaux ne sont pas permanents. L'un des participants souligne que l'Encyclopédie crée elle-même un certain patrimoine, qu'elle contribue à la « patrimonialisation » d'éléments de la culture. On propose de permettre aux jeunes d'identifier leur patrimoine (dans une perspective d'interactivité). On suggère aussi d'ajouter des notices biographiques sur les auteurs et de disséminer les informations rassemblées dans l'Encyclopédie dans divers réseaux d'échange présents sur la Toile tels que Facebook, YouTube et Wikipédia, afin d'accroître la visibilité et la fréquentation de l'Encyclopédie. 
II- Deuxième sujet de discussion : l'organisation des contenus

du site web de l'Encyclopédie

Présentation de Catherine Ferland pour l'Encyclopédie suivie des commentaires de l'expert invité Gilles Herman, directeur des Éditions du Septentrion.

A) Catherine Ferland expose la nouvelle grille thématique mise au point pour simplifier et pour rendre plus efficace la consultation de l'Encyclopédie par les internautes. Il s'agit d'une grille de six catégories subdivisées en une trentaine de sous-catégories. Cette grille a été développée à partir des catégories déjà assignées à la cinquantaine d'articles en ligne. Elle a été testée et s'avère pertinente pour clarifier le lien entre chacun des articles et le thème auquel il se rattache. Cette nouvelle grille facilitera la recherche exploratoire des articles à partir de divisions et sous-divisions thématiques courantes et assez générales pour inclure plusieurs articles chacune.

B) Gilles Herman souligne le piège de la catégorisation et souligne que cette opération n'est ni simple ni facile. Souvent, cette opération est plus utile pour les créateurs de contenus qui veulent organiser leur ouvrage que pour ceux qui les consultent. En effet, les internautes recourent de plus en plus à la fonction Recherche à partir d'un ou de quelques mots significatifs. Il insiste sur l'intérêt de localiser chaque sujet d'article dans le temps et dans l'espace et d'indexer tous les médias (son, image, texte). Il suggère également de maximiser l'interconnexion des contenus de l'Encyclopédie avec d'autres sites de la Toile, notamment par hyperliens. L'objectif étant de faire circuler l'information le plus possible, d'accroître la fréquentation de l'Encyclopédie et de diffuser largement son contenu. Il suggère enfin de créer quelques " chemins thématiques", des " histoires » de thèmes patrimoniaux qui lieraient plusieurs articles les uns aux autres et guideraient les utilisateurs le long d'une trame explicative, ou narrative.

La discussion qui suit permet de rappeler que la catégorisation est une démarche essentielle, car elle favorise le repérage des contenus par les moteurs de recherche de la Toile (notamment Google). La subdivision de la première catégorie "Individus et groupes » pose problème aux yeux de certains participants et devra être révisée. On explique que la rédaction attribue plus d'une catégorie ou sous-catégorie à chacun des articles afin de maximiser son repérage éventuel par les usagers. Les localisations temporelles (de type ligne du temps) doivent se faire par périodes, puisque la plupart des sujets couvrent une plage temporelle assez large. Même remarque pour la localisation géographique qui est parfois précise, et quelquefois « territoriale » (s'appliquant à de grandes étendues). Il faut aussi fournir des explications sur l'usage proposé d'un nouveau mode de présentation des contenus, soit celui d'un « nuage » de mots-clés thématiques, censé offrir aux usagers une 
porte d'entrée supplémentaire, plus intuitive. Ce nuage suggère en quelque sorte des contenus à explorer par les internautes, au lieu de s'en remettre à eux pour entrer les mots clés qui les guideront vers les articles liés à leurs centres d'intérêt. Les deux approches sont complémentaires. Le débat illustre clairement que le processus de catégorisation est complexe.

\section{III - Troisième sujet de discussion : la sélection des sujets et des documents multimédias complémentaires}

Présentation de Martin Fournier pour l'Encyclopédie suivie des commentaires de l'experte invitée Nathalie Hamel, consultante en ethnologie, patrimoine et muséologie.

A) Martin Fournier expose les contraintes auxquelles l'équipe de l'Encyclopédie fait face dans le recrutement d'auteurs pour couvrir des sujets précis, ainsi que dans le processus de révision qui conduit à la publication de la version finale des articles. En somme, le choix des sujets dépend toujours de la possibilité de trouver un auteur spécialiste disponible. Des sujets non prévus s'additionnent au calendrier de production quand l'Encyclopédie reçoit des propositions non sollicitées, mais dignes d'intérêt. Enfin, la qualité des articles ne peut pas toujours être optimisée autant que la direction le souhaiterait, compte tenu de la compétence variable des auteurs et de leur capacité, ou disponibilité plus ou moins grande à nous accompagner dans le processus de révision. L'accroissement du nombre de documents multimédias pertinents associés à chaque article est une avenue très favorable au développement de l'Encyclopédie, mais la sélection et le traitement de ces documents demande beaucoup de temps et d'énergie.

B) Nathalie Hamel fait remarquer que le choix des sujets comporte des lacunes au niveau des institutions et des acteurs marquants du patrimoine. Elle remarque également que les aléas de la production ont provoqué un retard dans la couverture de sujets incontournables du patrimoine de l'Amérique française, et, en contrepartie, la présence de sujets secondaires ou périphériques. Elle souligne le risque d'enlever de la crédibilité au projet si les principaux sujets d'importance ne sont pas couverts rapidement. À ce propos, elle se demande si le terme « encyclopédie » qui a une connotation d'exhaustivité sert bien le projet tel qu'il se réalise. Elle suggère de lancer des appels de textes ou encore de commander la rédaction de certains articles à des auteurs pigistes qui ne sont pas toujours des spécialistes reconnus des sujets à couvrir, mais qui pourraient effectuer une recherche de bon niveau et rédiger des articles tout à fait satisfaisants. Elle suggère enfin de définir certains concepts à l'intérieur de l'Encyclopédie et de préciser davantage l'orientation éditoriale. 
La discussion qui suit permet de préciser la démarche privilégiée par le directeur de l'Encyclopédie, Laurier Turgeon, qui en est une d'exploration des nouvelles tendances et manifestations du patrimoine. L'Encyclopédie est donc volontairement restée ouverte à divers points de vue et sujets touchant le patrimoine de l'Amérique française. Tous les participants jugent constructif, voire nécessaire, de publier sur le site de l'Encyclopédie une liste de sujets prioritaires qui n'ont pas encore trouvé d'auteur (de 30 à 40 sujets). Il est aussi proposé de chercher à engager davantage les institutions universitaires dans lesquelles travaillent les membres du comité scientifique, afin d'apporter un appui plus important et plus stable au recrutement d'auteurs et au développement du projet. La division de certains sujets majeurs en plus d'un article, afin d'en éclairer précisément toutes les facettes, telle que proposée par le coordonnateur de l'Encyclopédie est jugé pertinent, mais demande une grande vigilance. Il ne faut pas abuser de cette orientation.

\section{IV - Quatrième sujet de discussion : les processus de patrimonialisation}

Présentation de Laurier Turgeon pour l'Encyclopédie (sur support vidéo) suivie des commentaires de l'expert invité André Charbonneau, responsable des services historiques à Parcs Canada, Centre de services du Québec.

A) Laurier Turgeon expose divers processus de « mise en patrimoine» qui illustrent le travail des acteurs sociaux dans la sélection des éléments de la culture et de l'histoire qui seront mis de l'avant et présentés comme saillants à la collectivité, éventuellement mis en valeur, et formeront le patrimoine culturel. Ces choix ou ces préférences, conscients ou spontanés, individuels ou collectifs, ont souvent pour objectif de consolider le sentiment d'appartenance à une collectivité - l'identité - ou de garder présents certains événements du passé ou certaines valeurs propres à un groupe. Le terme « patrimonialisation » qui désigne ces processus est récent, tout autant que cette vision dynamique du patrimoine qui était autrefois perçu comme très stable. Le terme n'existe d'ailleurs pas encore en anglais. C'est notamment pourquoi l'Encyclopédie est un projet novateur et qu'il comporte une dimension exploratoire. L'Encyclopédie cherche à mieux faire comprendre cette notion de métissage du patrimoine à ses auteurs et à ses lecteurs, notamment en soulignant les apports interculturels qui enrichissent et modifient le patrimoine au fil du temps.

B) André Charbonneau présente le fonctionnement de quelques démarches très bien structurées mises en place par le gouvernement fédéral afin d'identifier les biens patrimoniaux dont il a la responsabilité. Par exemple, la collaboration du fédéral au programme international RAMSAR qui définit des aires de protection des milieux humides, le processus de reconnaissance 
de biens patrimoniaux propre à Parcs Canada, ou l'identification d'édifices appartenant au gouvernement fédéral dont la valeur patrimoniale nécessite des mesures de protection ou de mise en valeur. Ces démarches aboutissent à un classement hiérarchique et à des mesures appropriées à la valeur patrimoniale établie, selon de nombreux critères d'évaluation standardisés. Certaines de ces démarches sont entreprises par la population, d'autres par l'appareil gouvernemental.

La discussion qui suit témoigne de l'importance du concept de patrimonialisation dans le projet d'Encyclopédie, en même temps que de la difficulté de cerner cette notion précisément et de l'appliquer au traitement de tous les sujets. Le caractère exact du projet apparaît plus nettement aux yeux de certains participants à l'atelier. Ce débat met également en évidence la difficulté de délimiter trop précisément la notion de patrimoine qui demeure un objet vivant, en transformation, « comme un virus » suggère un participant. L'exploration de cette notion apparaît donc comme une contribution originale de l'Encyclopédie et une démarche scientifique caractéristique du projet.

Les échanges montrent bien que la problématique du patrimoine culturel francophone en Amérique du Nord mérite d'être étudiée avec attention, car le sujet s'avère plus complexe qu'on ne l'imaginait. À ce propos, Leslie Choquette attire l'attention sur la difficulté de traiter le patrimoine des FrancoAméricains sous l'angle de la patrimonialisation. Il en est de même, quoique de façon moins aigüe, du patrimoine des francophones en milieu minoritaire au Canada. En effet, qu'en est-il d'un patrimoine qui est sommairement mis en valeur, ou pas du tout? Comment cerner dans ces cas le processus de patrimonialisation? Au sujet d'un patrimoine franco-américain en particulier, le collège de l'Assomption, à Worcester, au Massachussets, il est suggéré que la démarche de création de ce collège francophone et, plus largement, d'institutions semblables, ainsi que la formation durable d'une élite francophone dans certains états de la Nouvelle-Angleterre, témoigne d'une volonté et d'actions concertées visant à maintenir une culture francoaméricaine et à créer une base patrimoniale durable aux États-Unis. La patrimonialisation serait alors de cet ordre. Le patrimoine n'est pas éternel. Il naît et il meurt. Il se transforme.

Comme le soulignait Laurier Turgeon dans son intervention vidéo, les auteurs de 1'Encyclopédie doivent être attentifs à plusieurs formes de patrimonialisation, qu'elle s'exprime par une reconnaissance ou un engouement populaire, ou qu'elle résulte d'une démarche de valorisation organisée et soutenue par une élite ou un pouvoir public. De toute façon, la description et l'analyse des processus de patrimonialisation éclairent les perceptions et les intentions des acteurs sociaux. 


\section{Principales retombées de la rencontre}

\section{À court terme}

La rencontre a permis de valider la deuxième phase de développement du site Internet de l'Encyclopédie, telle que soumise à la réflexion des participants à l'atelier. Les discussions ont mis en évidence les priorités absolues à réaliser (à l'intérieur du budget disponible) et souligné des détails qui avaient échappé jusqu'ici à l'équipe de production, comme la publication d'une liste de sujets prioritaires à couvrir et des améliorations à apporter à la liste des catégories. La version améliorée du site de l'Encyclopédie qui sera rendue disponible à l'été 2009 promet d'être pleinement satisfaisante. Il ressort des discussions qu'un projet de cet ordre - une encyclopédie diffusée sur la Toile - suppose une symbiose contenant-contenu plus étroite qu'une publication papier conventionnelle, étant donné la puissance de cette technologie, son évolution constante et rapide, ainsi que ses caractéristiques propres qui exercent une influence directe sur les modes de consultation de l'ouvrage par les usagers. En définitive, ce mode de consultation modifie le rapport des usagers à l'information contenue dans l'Encyclopédie.

\section{À moyen terme}

Nos discussions ont également permis d'identifier plusieurs éléments à mettre en place dans la troisième phase de développement du site Internet de l'Encyclopédie, en 2010. Par exemple, une localisation temporelle des sujets et une localisation spatiale plus fine et facilement accessible, une page d'accès aux contenus très détaillée, de brèves introductions à certains sujets et notions clés, un plan de large interconnexion et dissémination de l'Encyclopédie sur la Toile, des séries d'articles liés les uns aux autres par une trame logique ou narrative, l'ajout de notices biographiques d'auteurs, et autres.

\section{Prospective 2010}

Au moment de mettre sous presse, la diffusion d'un total de 225 articles en français et de 125 articles en anglais est assurée sur le site de l'Encyclopédie du patrimoine de l'Amérique française d'ici l'été 2010. 